\title{
-Original一
}

\section{Analysis of Alteration of Blood Pressure Response to Exercise through Baroreflex}

\author{
Hiromi Manabe, Nagaharu Fukuma, Takaya Tuchida, \\ Yuko Kato, Kosuke Mabuchi and Teruo Takano \\ Department of Functional Pathophysiology for Human Organs, Graduate School of Medicine, Nippon Medical School
}

\begin{abstract}
Background: The baroreflex has been reported to play an important role in hemodynamic regulation during exercise. Therefore, impairment of baroreflex function can induce an abnormal response of systolic blood pressure (SBP) to exercise, including exercise-induced hypertension. To clarify whether baroreflex function alters SBP response, we examined the relationship of baroreflex sensitivity (BRS) with SBP response to exercise.

Methods: In 22 subjects without cardiac dysfunction, BRS ( $\mathrm{ms} / \mathrm{mmHg}$ ) was measured by the phenylephrine method, and a treadmill exercise test was administered according to Bruce's protocol.

Results: 1) The chronotropic response to exercise was higher in the normal BRS group than in the reduced BRS group $(\mathrm{p}<0.01)$. The SBP at the initial phase of exercise $(1 \mathrm{~min}$ after the start of exercise) showed a smaller increase in the normal BRS group than in the reduced BRS group $(\mathrm{p}<0.01)$. During the initial phase of exercise, BRS had negative correlation with the SBP increment from rest $(r=-0.408, \mathrm{p}<0.05)$. During submaximal exercise $(6 \mathrm{~min}$ after the start of exercise), a positive correlation between BRS and SBP response $(r=0.422, p<0.05)$ was shown. 2) Subjects were divided into 2 groups: 12 subjects with normal BRS ( $\geq 5 \mathrm{~ms} / \mathrm{mmHg}$ ) and 10 subjects with reduced BRS $(<5 \mathrm{~ms} / \mathrm{mmHg})$. During the initial exercise phase, the negative correlation between BRS and SBP response was stronger in the normal BRS group $(r=-0.398)$ than in the reduced BRS group ( $r=-0.126)$. During submaximal exercise, BRS had a positive correlation with BP response to exercise in subjects with normal BRS ( $r=0.462)$.

Conclusion: Preserved baroreflex function is thought to be related to the pressor response to submaximal exercise, although the baroreflex is thought to be associated with the stabilization of blood pressure change during the initial exercise phase. These findings suggest that exercise-induced hypertension develops through the baroreflex mechanism.
\end{abstract}

(J Nippon Med Sch 2007; 74: 123-130)

Key words: baroreflex, blood pressure, exercise, hypertension

Correspondence to Hiromi Manabe, MD, Department of Functional Pathophysiology for Human Organs, Graduate School of Medicine, Nippon Medical School, 1-1-5 Sendagi, Bunkyo-ku, Tokyo 113-8603, Japan

E-mail: hiromi-manabe@nms.ac.jp

Journal Website (http://www.nms.ac.jp/jnms/) 


\section{Introduction}

Clinical studies have indicated that an excessive response of blood pressure $(\mathrm{BP})$ to exercise predicts future hypertension ${ }^{1-3}$. This suggests that maladaptation of the cardiovascular system to exercise stress is related to the pathogenesis of hypertension. However, the mechanism of this association has not been confirmed, although an augmented sympathetic response to $\operatorname{exercise}^{4-6}$ has been discussed.

Lately, the baroreflex mechanism as a cause of hypertension has attracted attention. In past investigations, reduced baroreflex function has been reported to be associated with the development of hypertension by allowing incremental increases in $\mathrm{BP}^{7-9}$. This pathway to the development of hypertension is considered to indicate a passive effect of baroreflex on BP regulation. In most newer reports, the baroreflex mechanism has been suggested to work aggressively to maintain high BP by shifting the operating point to a higher pressure with sympathetic excitation. For example, the baroreflex is reported to play an important role in hemodynamic regulation during exercise while shifting the baroreflex operating point higher via central commands, ergoreflex or metaboreflex or both $^{10-14}$. It has been speculated that an exaggerated response to stimulation through the baroreflex mechanism leads to development of hypertension. These findings indicate that hypertension can be induced by either hypofunction or hyperfunction of the baroreflex, but this has not been confirmed clinically.

The aim of the present investigation was to examine the influence of baroreceptor reflex sensitivity (BRS) on response of $\mathrm{BP}$ to exercise and to investigate the possible role of the baroreflex mechanism in the development of hypertension suggested by previous reports. Therefore, we studied the relationship of BRS and the systolic BP (SBP) response to exercise. Assessment was based on comparisons of SBP and impaired and normal baroreflex function between two exercise phases, which were the initial ( $1 \mathrm{~min}$ after the beginning of exercise) and submaximal (6 min after the beginning of exercise) exercise phases.

\section{Materials and Methods}

\section{Study Population}

The study population consisted of 22 subjects (7 women and 15 men; mean age, $62 \pm 8$ years [mean \pm $\mathrm{SD}])$ who consecutively visited our Department of Cardiology. They underwent transthoracic echocardiography, treadmill exercise testing, and testing for BRS. Subjects were eligible for the study if exercise was only limited by symptoms of fatigue or dyspnea but not by angina, syncope, or claudication. Excluded were patients who had had a myocardial infarction within 3 months before the study, unstable angina, left ventricular systolic dysfunction (left ventricular ejection fraction $<50 \%$ ), marked resting hypertension $(\geq 180 / 110 \mathrm{mmHg}$ ), diabetes mellitus with neuropathy or were 70 years or older. In patients with ischemic heart disease, the diseased coronary artery had been treated. Other exclusion criteria were the inability to reach stage II in the standard Bruce protocol and the use of $\beta$ blockers or non-dihydropyridine calcium-channel blockers, such as diltiazem and verapamil. The reason for these exclusion criteria is that these factors were likely to influence results of exercise testing. All aspects of the study were carefully explained to study subjects before informed consent for participation was given. These subjects were divided into 2 groups: a normal BRS group comprising 12 subjects with BRS of $5 \mathrm{~ms} / \mathrm{mmHg}$ or more and a reduced BRS group comprising 10 subjects with BRS of less than $5 \mathrm{~ms} / \mathrm{mmHg}$.

\section{BRS}

BRS was assessed by the bolus phenylephrine method of Smyth et al. ${ }^{15}$ Subjects were placed in a supine position in a comfortable environment for at least 30 minutes. Electrocardiograms were monitored by continuous recording of lead II. The $\mathrm{BP}$ was measured from the radial artery on the side opposite the intravenous line using the Jentow noninvasive continuous BP monitoring apparatus (Nihon Colin Co., Ltd., Aichi, Japan) during the entire 
Alteration of BP Regulation by Baroreflex

Table 1 Comparison of background between normal and reduced BRS groups

\begin{tabular}{lccc}
\hline & Normal BRS & Reduced BRS & p-value \\
\hline Age (years) & $59 \pm 14$ & $63 \pm 7$ & $\mathrm{~ns}$ \\
Sex (male : female) & $9: 3$ & $6: 4$ & $\mathrm{~ns}$ \\
Diabetes mellitus & $4 / 12(33 \%)$ & $3 / 10(30 \%)$ & $\mathrm{ns}$ \\
Hypertension & $3 / 12(25 \%)$ & $3 / 10(30 \%)$ & $\mathrm{ns}$ \\
Previous CAD & $4 / 12(33 \%)$ & $3 / 10(30 \%)$ & $\mathrm{ns}$ \\
Medications & & & \\
$\quad$ ACEI and/or ARB & $3 / 12(25 \%)$ & $3 / 10(30 \%)$ & $\mathrm{ns}$ \\
\hline
\end{tabular}

Data are expressed as mean $\pm \mathrm{SD}$; P value was calculated between normal and reduced BRS group; ns, not statistically significant; CAD, coronary artery disease; ACEI, angiotensin-converting enzyme inhibitors; ARB, angiotensin II receptor blockers. Hypertension is defined as a $\mathrm{BP}$ of $150 / 90 \mathrm{mmHg}$ or greater. Characteristics shown in the table did not differ between the normal and reduced BRS groups.

test. After the stabilization period, patients received bolus administration of phenylephrine $(2 \mu \mathrm{g} / \mathrm{kg}$ IV) to increase SBP by 15 to $40 \mathrm{mmHg}$; if the desired increase was not achieved, the dose was increased to $3 \mu \mathrm{g} / \mathrm{kg}$. The bolus injection was repeated at least 3 times at whatever dose was found to be efficacious. Then the linear regressions of the R-R intervals on the electrocardiogram and the SBP were calculated, including all points between the beginning of the first significant increase in systolic arterial pressure and the end of the plateau of systolic arterial pressure. If the correlation coefficients were statistically significant $(r \geq 0.6, p<$ $0.05)$, test results were used for analysis. The final slope of these regression lines was the mean value of at least 3 tests and was considered to be an index of BRS, which was expressed in milliseconds per $\mathrm{mmHg}$ (ms/mmHg). To define an abnormal BRS, the BRS of healthy subjects was analyzed in our previous study. A value $(<5 \mathrm{~ms} / \mathrm{mmHg})$ below the mean BRS minus $2 \mathrm{SD}$ in healthy subjects was defined as abnormally depressed. This value indicating abnormality is similar to those previously reported.

\section{Exercise Treadmill Testing}

All patients underwent symptom-limited treadmill exercise testing according to the standard Bruce protocol ${ }^{16}$. Exercise was stopped by symptoms of fatigue or dyspnea when patients reached a rating on the Borg perceived exertion scale of $17^{17}$. Heart rate and 12-lead electrocardiogram were monitored continuously during exercise using the Case 15 Stress System (Marquette Electronics, Inc., Milwaukee, WI, USA). BP was measured every minute with an automatic sphygmomanometer (STBD-780B, Nihon Collin Co., Ltd.), which is commonly used in Japan and is considered to be reliable because it detects the Korotokoff sound supported by the ECG signal. Exercise capacity in metabolic equivalents (METs) was estimated using the formula described by the American College of Sports Medicine ${ }^{18}$.

Arterial BP was compared at submaximal exercise. Evaluation of BP response to peak exercise is inadequate for determining the influence of the baroreflex on $\mathrm{BP}$ response because $\mathrm{BP}$ response to intense exercise is thought to be affected mainly by mechanisms other than the baroreflex.

\section{Statistical Analysis}

All statistical analyses were peformed with the SPSS program (SPSS, Inc., Chicago, IL, USA), and all values are expressed as mean \pm SD. We investigated the relationship between BRS and the exercise SBP response during the initial exercise phase and the submaximal phase. Comparisons of variables between groups were performed using the unpaired Student's $t$-test and chi-square analysis. Analysis of correlation between values was performed with Pearson's method. Differences were considered statistically significant at $\mathrm{p}$ values less than 0.05 . 
Table 2 Difference in response to treadmill exercise between the normal BRS and reduced BRS groups

\begin{tabular}{lccc}
\hline & Normal BRS & Reduced BRS & p-value \\
\hline Workload (METs) & $9.3 \pm 3.0$ & $8.2 \pm 2.1$ & $\mathrm{~ns}$ \\
Heart rate (beat/min) & & & \\
at rest & $78 \pm 9$ & $70 \pm 10$ & $\mathrm{~ns}$ \\
at peak exercise & $149 \pm 12$ & $121 \pm 18$ & $<0.01$ \\
SBP (mmHg) & & & \\
at rest & $133 \pm 13$ & $144 \pm 19$ & $\mathrm{~ns}$ \\
at 1 min after starting & $140 \pm 13$ & $157 \pm 11$ & $<0.01$ \\
at 6 min after starting & $174 \pm 21$ & $178 \pm 13$ & $\mathrm{~ns}$ \\
at peak exercise & $191 \pm 23$ & $200 \pm 20$ & $\mathrm{~ns}$ \\
\hline
\end{tabular}

$\mathrm{P}$ value was calculated between normal and reduced BRS group; ns, not statistically significant; METs, metabolic equivalent. The reduced BRS group showed an impaired chronotropic response to exercise, and BP was significantly increased in the initial phase of exercise in comparison with the normal BRS group.

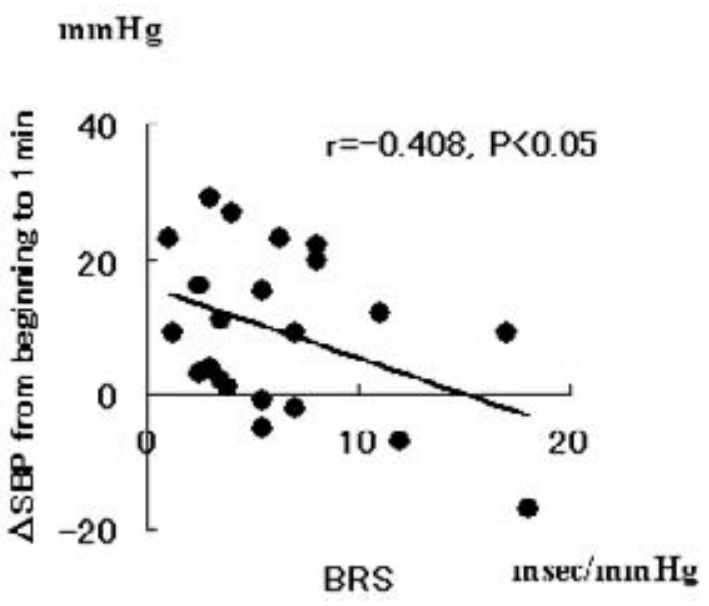

Fig. 1 BRS and exercise SBP in the initial phase of exercise

$\triangle \mathrm{SBP}$ : increase in SBP from rest to $1 \mathrm{~min}$ after the beginning of exercise. In the initial phase of exercise (at $1 \mathrm{~min}$ ), BRS was negatively correlated with the increase in SBP from rest $(r=-0.408, \mathrm{P}<0.05)$.

\section{Results}

As shown in Table 1, age, sex, and the presence of diabetes mellitus and hypertension (>150/90 $\mathrm{mmHg}$ ) did not differ between the normal and reduced BRS groups. Subjects with reduced BRS showed chronotropic incompetence on treadmill exercise testing. The BPs at rest, $6 \mathrm{~min}$ after starting exercise, and at peak exercise did not differ between the two groups, and the $\mathrm{BP}$ response at 1

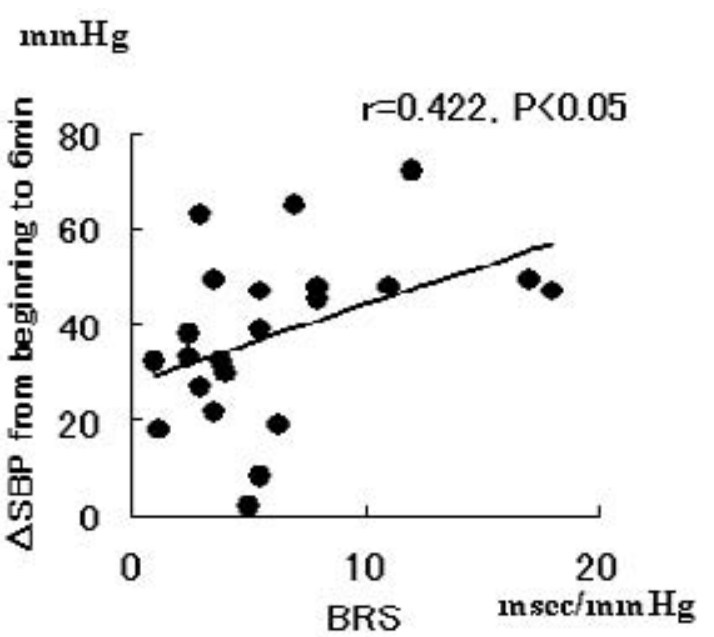

Fig. 2 BRS and exercise SBP in the submaximal phase of exercise

$\triangle \mathrm{SBP}$ : increase in SBP from rest to $6 \mathrm{~min}$ after the beginning of exercise. In the submaximal phase of exercise (at $6 \mathrm{~min}$ ), BRS had a positive correlation with the increase in SBP from rest $(\mathrm{r}=0.422, \mathrm{P}<0.05)$.

min after the start in the normal BRS group showed a significant suppression of increases in comparison with the reduced BRS group (Table 2).

The association between BRS and the response of SBP to exercise was examined for initial and submaximal exercise. In only 5 of the 22 subjects, SBP in the initial exercise phase decreased in comparison with that at rest. In the initial exercise phase, the relationship of the increase of the BP response correlated negatively with BRS (Fig. 1). 
Table 3 Correlation coefficient between BRS and SBP

\begin{tabular}{ccc}
\hline & Normal BRS & Reduced BRS \\
\hline Correlation coefficient of BRS with SBP & \\
at 1 min after starting & $\mathrm{r}=-0.398$ & $\mathrm{r}=-0.126$ \\
at 6 min after starting & $\mathrm{r}=0.462$ & $\mathrm{r}=0.231$ \\
\hline
\end{tabular}

The correlation coefficient between BRS and BP response indicated the tendency for $\mathrm{BP}$ response to be greater in subjects with normal BRS than in subjects with reduced BRS, but the difference was not statistically significant.

Especially in subjects with normal BRS function, that is, with preserved BRS function, just after the beginning of exercise BP can decrease to less than that at rest. In submaximal exercise, however, increases in SBP from rest to 6 min after the start of exercise was positively correlated with BRS (Fig. 2).

To clarify further the influence of BRS on BP response to exercise, our study population was divided on the basis of BRS. Table 3 shows the difference in the correlation coefficient of BRS with $\mathrm{BP}$ response between the normal and reduced BRS groups. In subjects with preserved baroreflex function, the correlation between BRS and BP response both at initial and submaximal exercise was stronger than in subjects with reduced function, but the difference between groups was not statistically significant.

\section{Discussion}

The present study assessed the influence of the baroreflex system on the response of arterial BP to exercise. The results suggested that the association of the baroreflex with the BP response is suppressive in the initial phase of exercise and is accelerative at submaximal exercise, especially in cases of preserved baroreflex function. The exerciseinduced BP elevation did not relate to a reduction of baroreflex function, but rather to the preservation of baroreflex function. This phenomenon is useful for understanding the mechanism of exercise-induced hypertension.

\section{BP Control through Baroreflex Mechanism in} the Initial Phase of Exercise

Previously, we reported the correlation between
BRS and exercise heart rate response ${ }^{19.20}$. In these reports, we showed baroreflex-related heart rate responses to exercise in both the initial and submaximal phases of exercise through parasympathetic and sympathetic means. The present study similarly indicated an association between BRS and the heart rate response. Concerning arterial $\mathrm{BP}$, however, the response to exercise differed among exercise intensities. The reason for these differences may be that from the standpoint of the autonomic system, the BP response might be affected mainly by sympathetic stimulation rather than by a parasympathetic effect.

The present investigation showed that BRS has a negative correlation with the increase in SBP from rest to $1 \mathrm{~min}$ after the beginning of exercise. A similar phenomenon was reported by Melcher et al. ${ }^{13}$ in which arterial and cardiopulmonary baroreceptors have a major role in suppressing changes in arterial pressure at the start of exercise. In particular, they reported that a decrease in SBP in the early phase of exercise was prevented by BRS. In the present study, however, most subjects exhibited an elevation in SBP as an initial exercise response. A possible reason for this difference is that our population included subjects with hypertension or diabetes mellitus or both and was older. In the normal BRS group the change in SBP was restrained, suggesting that the preserved BRS acts to suppress changes in arterial BP in the initial phase, regardless of elevation or depression.

Concerning the role of the central command in hemodynamic regulation during the initial exercise phase, it has been reported that this mechanism leads to a shift of the operating point of the baroreflex to a higher pressure in proportion to 
work intensity and stimulates sympathetic activity $^{10,21,22}$. However, because of insufficient intervention of ergoreflexes in the initial phase, the shift of the set point of the baroreflex is incomplete and results in a weak response of SBP through the sympathetic system. In the initial exercise phase, therefore, the preserved baroreflex system is thought to be able to play a suppressive role in the regulation of SBP, which is similar to our present results.

\section{BP Control through Baroreflex Mechanism in} the Submaximal Exercise Phase

The present results indicated that during the submaximal phase, BRS had a positive correlation with the SBP response, especially in subjects with preserved baroreflex function. This finding suggests that in the submaximal phase, the baroreflex system performs an important role in the acceleration of sympathetic activity and that the preserved function of the baroreflex is required for adequate sympathetic support to adapt to stress.

The initial study in this field reported that BRS weakened as exercise intensity increased ${ }^{11}$. However, the baroreflex was later reported to regulate hemodynamics during exercise, shifting the set point of the target $\mathrm{BP}$ to a higher level ${ }^{12,23}$. To continue performance of intensive exercise, precisely sensing changes in the metabolic and mechanical environment through the baroreflex system is necessary to ensure sufficient blood flow to motor muscles during exercise. If $\mathrm{BP}$ does not reach the level demanded by working muscles, the baroreflex system leads to the activation of sympathetic tone $\mathrm{e}^{11,23}$ and $\mathrm{BP}$ elevation through the mechanoreflex and metaboreflex ${ }^{10,21,22}$. However, these findings have not been confirmed in a clinical study.

This present report attempted to clarify the roles of the baroreflex mechanism in the regulation of the $\mathrm{BP}$ response to submaximal exercise stress in human subjects, although the methodology was indirect. We found that in subjects with preserved baroreflex function, BRS had an accelerative relationship with the $\mathrm{BP}$ response. Our extensive review of the literature revealed that this is the first report of clinical evidence of baroreflex-induced presser effects during exercise.

\section{Impairment of Baroreflex Function as a Possible Cause of Hypertension}

A key report with regard to the development of hypertension through a reduction of $\mathrm{BRS}^{8}$ showed that the decrease of BRS is revealed in the phase before the development of hypertension. This finding indicates that the baroreflex function at rest relates to the pathogenesis of hypertension. In a newer investigation, however, subjects with mild borderline hypertension had a set point shift to a higher pressure level, although BRS remained in the normal range ${ }^{24}$. Similarly, in the present paper the attenuation of BRS at rest was not related to the development of hypertension or to the enhanced response of $\mathrm{BP}$ to exercise. These findings suggest that the development of hypertension require both a reduction in BRS and an additional mechanism, such as an increase in humoral factors that have a role in increases in peripheral vessel resistance ${ }^{25.26}$.

It has been reported ${ }^{24}$ that preserved baroreflex function and the shifting of the operating point to the higher $\mathrm{BP}$ is required for the development of hypertension. For example, the baroreflex system with an abnormally high operating set point is thought to stimulate the renin-angiotensin system through activation of the renal sympathetic nerve. In our subjects with preserved BRS, baroreflex function was correlated with the arterial BP response to exercise more strongly than in subjects with decreased BRS. In subjects with a hypersensitive baroreflex, i.e., greater than $10 \mathrm{~ms} /$ $\mathrm{mmHg}$ BRS, the SBP response was higher than in other subjects. Therefore, both sufficient baroreflex function and excess shifting of the operating point to the range of hypertension might lead to exerciseinduced hypertension, although our present results can only provide the basis for speculation.

To our knowledge, this is the first clinical report showing that the arterial BP response to exercise is altered by BRS and that this relationship is influenced by exercise intensity. Although these findings do not directly confirm the cause of hypertension through the baroreflex mechanism, they suggest a possible mechanism leading to the 
development of hypertension. In future, we must investigate the questions raised by our results. Also research into treatment for baroreflex abnormalities is needed. Several reports ${ }^{27-29}$ have suggested pharmacological and exercise therapies as methods to improve decreased BRS. However, therapy for the excess shifting of the operating point is still unknown. If such functions of the baroreflex could be recovered, adequate adaptation of the cardiovascular system to stress and a decrease in the incidence of hypertension may be achieved.

\section{Limitations}

We are aware of two limitations of the present work. The first concerns the study population. We did not collect data on patients with exerciseinduced hypertension, although some subjects did have excessive response of BP to exercise. However, this study is not clinical research on hypertension. We did speculate on the alteration of $\mathrm{BP}$ regulation during exercise by the baroreflex mechanism. To confirm the cause of hypertension from a pathophysiological basis, a more direct investigation should be done. However, we believe that the present study has clinical implications, because it is the first investigation of exercise-induced hypertension that concerns the baroreflex mechanism.

The second limitation is that baroreflex function was estimated at rest, but not during exercise. Because noninvasive and continuous measurement of arterial BP during dynamic exercise is difficult, such an evaluation is beyond the scope of this research. However, if the set point shift were to be directly examined, we could better address questions regarding the pathogenesis of hypertension via the baroreflex mechanism. In future, we should study not only BRS, but also the abnormal response of resetting the baroreflex to a higher systemic BP as an adaptation to stress.

\section{References}

1. Dlin RA, Hanne N, Silverberg DS, Bar OO: Follow-up of normotensive men with exaggerated blood pressure response to exercise. Am Heart J 1983; 106: 316-320.

2. Wilson NV, Meyer BM: Early prediction of hypertension using exercise blood pressure. Prev Med 1981; 10: 62-68.

3. Benbasaat J, Froom P: Blood pressure response to exercise as a predictor of hypertension. Arch Internal Med 1986; 146: 2053-2055.

4. Goldstein DS: Plasma norepinephrine during stress in essential hypertension. Hypertension 1981; 3: 551556.

5. Philipp TH, Distler A, Cordes U: Sympathetic nervous system and blood-pressure control in essential hypertension. Lancet 1978; 2: 959-963.

6. Miki K, Yoshimoto M, Tanimizu M: Acute shifts of baroreflex control of renal sympathetic nerve activity induced by treadmill exercise in rats. J Physiol 2003; 548: 313-322.

7. Ookuwa H, Takata S, Ogawa J, Iwase N, Ikeda T, Hattori N: Abnormal cardiopulmonary baroreflexes in normotensive young subjects with a family history of essential hypertension. J Clin Hypertension 1987; 3: 596-603.

8. Takeshita A, Tanaka S, Kuroiwa A, Nakamura M: Reduced Baroreceptor Sensitivity in Borderline Hypertension. Circulation 1975; 51: 738-742.

9. Volpe M, Trimarco B, Ricciardelli B, et al.: The automatic nervous tone abnormalities in the genesis of the impaired baroreflex responsiveness in borderline hypertensive subjects. Clin Sci 1982; 62: 581-588.

10. Potts JT, Mitchell JH: Rapid resetting of carotid baroreceptor reflex by afferent input from muscle receptors. Am J Physiol 1998; 275: H2000-H2008.

11. Walgenbach SC, Donald DE: Inhibition by carotid baroreflex of exercise induced increase in arterial pressure. Circ Res 1983; 52: 253-262.

12. Ichinose M, Saito M, Kondo N, Nishiyasu T: Timedependent modulation of arterial baroreflex control of muscle sympathetic nerve activity during isometric exercise in humans. Am J Physiol Heart Circ Physiol 2006; 290: H1419-H1426.

13. Melcher A, David ED: Maintained ability of carotid baroreflex to regulate arterial pressure during exercise. Am J Physiol 1981; 241: H838-H849.

14. Norton KH, Boushel S, Strange B, Saltin B, Raven PB: Resetting of the carotid arterial baroreflex during dynamic exercise in human. J Appl Physiol 1999; 87: 332-338.

15. Smyth HS, Sleight P, Pickering GW: Reflex regulation of arterial pressure during sleep in man:a quantitative method of assessing baroreflex sensitivity. Circ Res 1969; 24: 109-121.

16. Sheffield LT: Graded exercise tests for ischemic heart disease. In Exercise testing and training of apparently healthy individuals. A handbook for physicians, 1975; pp 35-38, American Heart association, Dallas, Texas.

17. Borg GA: Psychophysical bases of perceived exertion. Med Sci Sports Exerc 1982; 14: 377-381.

18. American College of Sports Medicine. Guidelines for Exercise Testing and Prescription. 3rd edition, 1986; p26, Lea \& Febiger, Philadelphia, PA.

19. Fukuma N, Oikawa K, Aisu N, et al.: Impaired baroreflex as a cause of chronotropic incompetence 
during exercise via autonomic mechanism in patients with heart disease. Int J of Cardiol 2004; 97: 503-508.

20. Fukuma N, Tuchida T, Ushijima A, et al.: Heart rate response to exercise and sympathetic excitability through the Baroreflex Mechanism. AHA : Circulation (supple28887); 2005; 281.

21. Raven PB, Fadel PJ, Ogoh S: Arterial baroreflex resetting during exercise: a current perspective. Exp Physiol 2006; 91: 37-49.

22. Smith SA, Querry RG, Fadel PJ, et al.: Partial blockade of skeletal muscle somatosensory afferents attenuates baroreflex resetting during exercise in human. J Physiol 2003; 551: 1013-1021.

23. Rowell LB, O'Leary DS: Reflex control of the circulation during exercise: Chemoreflex and Mechanoreflexes. J Appl Physiol 1990; 69: 407-418.

24. Eckberg DL: Carotid baroreflex function in young men with borderline blood pressure elevation. Circulation 1979; 59: 632-636.

25. Ponikowski P, Chua TP, Piepoli M, et al.: Augumented peripheral chemosensitivity as a potential input to baroreflex impairment and autonomic imbalance in chronic heart failure.
Circulation 1997; 96: 2586-2594.

26. Ichinose M, Saito M, Wada H, Kitano A, Kondo N, Nishiyasu T: Modulation of arterial baroreflex dynamic response during muscle metaboreflex activation in humans. J Physiol 2002; 544: 939-948.

27. Hill SS Jr, Vanoli E, Adamson PB, Verrier RL, Foreman RD, Schwartz PJ: Exercise Training Confers Anticipatory Protection From Sudden Death During Acute Myocardial Ischemia. Circulation 1994; 89: $548-552$.

28. Leitch JW, Newling RP, Basta M, Inder K, Dear K, Fletcher PJ: Randomized trial of a hospital-based exercise training program after acute myocardial infarction: cardiac autonomic effects. J Am Coll Cardiol 1997; 29: 1263-1268.

29. Iellamo F, Legramante JM, Massaro M, Raimondi G, Galante A: Effects of a Residential Exercise Training on Baroreflex Sensitivity and Heart Rate Variability in Patients With Coronary Artery Disease. Circulation 2000; 102: 2588-2592.

(Received, December 18, 2006)

(Accepted, January 6, 2007) 